OPEN ACCESS

Edited by:

George Bertsias,

University of Crete, Greece

Reviewed by:

Konstantinos Kambas,

Hellenic Pasteur Institut, Greece

Yohei Kirino,

Yokohama City University, Japan

*Correspondence:

Paolo Sfriso

paolo.sfriso@unipd.it

TORCID:

Paolo Sfriso

orcid.org/0000-0001-6432-7174

Specialty section:

This article was submitted to Rheumatology,

a section of the journal

Frontiers in Medicine

Received: 22 January 2020

Accepted: 14 April 2020

Published: 30 April 2020

Citation:

Oliviero F, Bindoli S, Scanu A, Feist E,

Doria A, Galozzi P and Sfriso P (2020)

Autoinflammatory Mechanisms in

Crystal-Induced Arthritis.

Front. Med. 7:166.

doi: 10.3389/fmed.2020.00166

\section{Autoinflammatory Mechanisms in Crystal-Induced Arthritis}

\author{
Francesca Oliviero ${ }^{1}$, Sara Bindoli ${ }^{1}$, Anna Scanu ${ }^{1}$, Eugen Feist ${ }^{2}$, Andrea Doria ${ }^{1}$, \\ Paola Galozzi ${ }^{1}$ and Paolo Sfriso ${ }^{1 *+}$ \\ ${ }^{1}$ Rheumatology Unit, Department of Medicine-DIMED, University of Padova, Padova, Italy, ${ }^{2}$ Department of Rheumatology, \\ Cooperation Partner of the Otto-von-Guericke, University Magdeburg, Helios Clinic, Vogelsang-Gommern, Germany
}

Crystal-induced arthritides have been classified as "type-1 autoinflammatory diseases" for their main features which resemble those of the monogenic autoinflammatory syndromes. They are in fact characterized by spontaneous onset, recurrence of the episodes, self-limitation and resolution, inflammasome activation with huge production of IL-1 $\beta$ and a prevalent involvement of the innate immune system. The term "auto" refers also to the induction of IL-1 $\beta$ gene expression, processing and secretion by IL- $1 \beta$ itself. The concept of autoinflammation in crystal-induced arthritis has been finally reinforced by the efficacy of IL-1 blockade in treating acute and chronic state of this disease. The aim of this article is to review the autoinflammatory mechanisms in crystal-induced arthritis, considering both clinical and molecular aspects.

Keywords: crystal-induced arthritis, interleukin-1, autoinflammatory disease, interleukin-1 receptor antagonist, inflammasome

\section{INTRODUCTION}

Crystal-induced arthritis (CIA) comprises two main inflammatory arthropathies, gout, and pseudogout, characterized by the formation and deposition in joints of monosodium urate (MSU) and calcium pyrophosphate (CPP) crystals, respectively. A third type of crystals, named basic calcium phosphate (BCP) crystals are associated with calcific periarthritis, osteoarthritis, and destructive arthropathies. The main features of CIA resemble those of the monogenic autoinflammatory syndromes such as: spontaneous onset, recurrence of the episodes, selflimitation and resolution, inflammasome activation with huge IL-1 $\beta$ production with prevalent involvement of the innate immunity (1). Although no specific genetic causes, but rather multigenetic predispositions have been associated with CIA, gout and pseudogout have been classified as "type-1 autoinflammatory diseases" (2).

The initial observation that uric acid is able to stimulate the innate immune system dates to 2003 when it was identified as a major endogenous danger signal released from injured cells capable to activate T-cell responses (3).

Two following works established the importance of IL-1 $\beta$ in CIA and laid the foundation for the definition of gout as autoinflammatory disease. The first concerned the demonstration that uric acid crystals (likely together with additional triggers) were able to activate the cytoplasmic NACHTLRRPYD-containing protein-3 (NLRP3) inflammasome which, through the caspase-1 activation, induced cleavage of pro-IL-1 $\beta$ to IL-1 $\beta$ (4). The second, which was conducted simultaneously, demonstrated the crucial role of IL-1 receptor and MyD88 molecule in non-myeloid derived cells, in triggering and amplify the inflammatory response induced by pathogenic crystals (5).

The prefix "auto" in autoinflammation does not refer only to the spontaneous resolution, but also implies that IL-1 induces its own gene expression, processing and secretion (6), and therefore, 
the chronic triggering of IL- 1 receptor by IL- $1 \beta$ itself can be considered as an autoinflammatory process (7).

The efficacy of IL-1 blockade in treating acute and chronic crystal arthritis has finally reinforced the concept of autoinflammation in those diseases $(8,9)$.

The aim of this article is to review the autoinflammatory mechanisms in CIA, considering both the molecular and clinical aspects.

\section{CLINICAL COURSE}

The recurrent and apparently not-provoked episodes of CIA are typical of autoinflammatory diseases. Both gout and pseudogout are characterized by an acute and a chronic phase. The first phase is characterized by recurrent acute attacks that resolve spontaneously over a period of 7 to 10 days, with asymptomatic periods between attacks.

The onset of the acute attack is abrupt, with symptoms and signs of severe acute inflammation (intense pain, redness, warmth, swelling, tenderness, and joint disability). If untreated, acute attack in gouty patients commonly resolves within a few days, while initial episodes of acute CPP crystal arthritis may persist longer (1 to 3 weeks) before remitting (10). Some patients may experience one single attack, but more often other episodes occur within 6 months to 2 years. Chronic tophaceous gout with severe joint destruction (Figure 1A) can also develop after many years of recurrent polyarticular gout. The deposition of BCP crystals leads to different clinical manifestations, the most severe of which are calcific periarthritis and the destructive arthropathy of the elderly known as Milwaukee shoulder (or knee) syndrome. While acute calcific periarthritis is characterized by a painful onset and a self-limiting course, Milwaukee shoulder syndrome is characterized by intermittent pain and large noninflammatory effusions.

As regards affected joints, the first metatarsophalangeal joint is the most frequently affected in gout, the knee is affected in over 50 percent of all acute attacks of acute CPP crystal arthritis (Figure 1B) (11). Other joints such as the wrists, shoulders, ankles and hands can also be affected by CIA.

Gout and pseudogout may also present different extraarticular manifestations such as hypertension, obesity and dyslipidemia, which make these patients at higher risk to develop cardiovascular comorbidities and renal dysfunctions.

\section{FEVER}

The activation of NLRP3 inflammasome by crystals, which determines the final production of IL- $1 \beta$, may be considered a possible mechanism for the onset of fever in patients affected by crystal arthropathies. Although systemic fever occurs in a minority of subjects with acute gout, it may be present in patients with repeated polyarticular attacks (12). As reported in literature in the past (13), fever may be more prevalent (up to 50\%) in those affected by CPP crystal-induced arthritis than in gouty patients. Similar cases were reported by Berger et al. who observed the presence of fever in association with high sedimentation rate
(ESR) in subjects affected by CPP deposition (14). Overall, CIA may be often the cause of febrile systemic inflammatory diseases particularly in elderly population (15). However, it is important to recognize when febrile episodes are related to infectious arthritis or a systemic sepsis. Therefore, in order to avoid misdiagnosis and uncorrected therapies, it is suggested to perform additional laboratory and microbiological tests to confirm the diagnosis. In this context, synovial fluid analysis is the most specific diagnostic test in presence of joint effusion, and it should be always performed in case of acute monoarthritis.

It was observed that in patients with chronic tophaceous gout the polyarticular repeated attacks may induce a systemic inflammatory response syndrome (SIRS) without an associated infection (16). Similarly, the uncommon axial involvement in polyarticular gout has the capability to induce a SIRS reactionlike mimicking a sepsis within the context of a chronic crystal arthropathy (17). Likewise, the crowned dens syndrome, due to calcium pyrophosphate or hydroxyapatite deposition in the cervical spine around the odontoid process (Figure 1C), may present with severe occipital pain, neck stiffness and high fever and can be often misdiagnosed as polymyalgia rheumatica or meningitis (18). In general, the prevalence of fever in crystalinduces arthropathies is driven by specific pyrogens (IL-1, IL6 , TNF- $\alpha$ ) with the inflammasome as pivotal activator of the inflammation cascade.

\section{ENDOGENOUS AND EXOGENOUS FACTORS INVOLVED IN TRIGGERING CRYSTAL-INDUCED INFLAMMATION}

Studying the molecular mechanisms of CIA gave notable details about the NLRP3 inflammasome and the release of proinflammatory cytokines (4). Nevertheless, it is important to bear in mind that hyperuricemia can be asymptomatic, and crystals can be present without an inflammatory response, suggesting the existence of regulatory mechanisms that can modify the acute inflammatory response (19).

Recent discoveries light up the complex associations of endogenous and exogenous factors at the initial step of the inflammatory inflammasome-mediated process (Figure 2). The canonical response of the inflammasome to the "second signal," granted by the interaction of crystals with inflammasomecompetent cells, requires "first signal" priming events, in order to avoid inappropriate firing of the pathway (20). The mechanisms by which MSU crystals trigger the "second signal" are still poorly understood. Perturbation in intracellular ionic homeostasis, especially potassium and calcium balance, leads to mitochondrial reactive oxygen species (ROS) generation before assembling the inflammasome (21).

Different conditions promoting an underlining inflammatory response can lead to a non specific "first signal" priming step. Endogenous factors include the complement protein C5a, the granulocyte-macrophage colony-stimulating factor (GM-CSF), the heterodimer S100A8/A9, and the protein fraction of synovial fluids. Exogenous factors include long chain saturated fatty acids and spikes in systemic levels of acetate. 


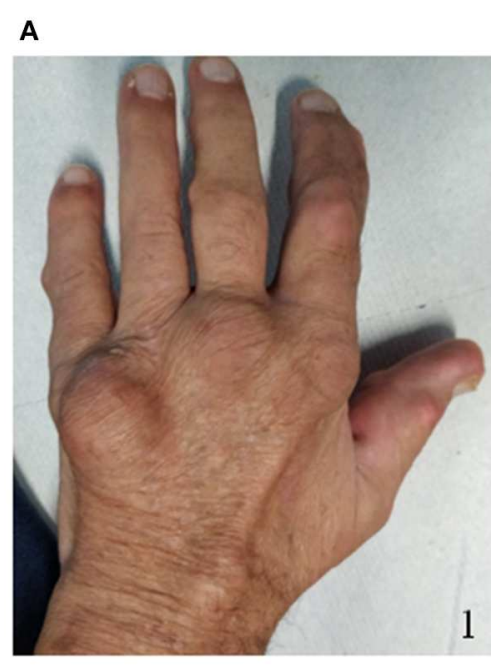

B

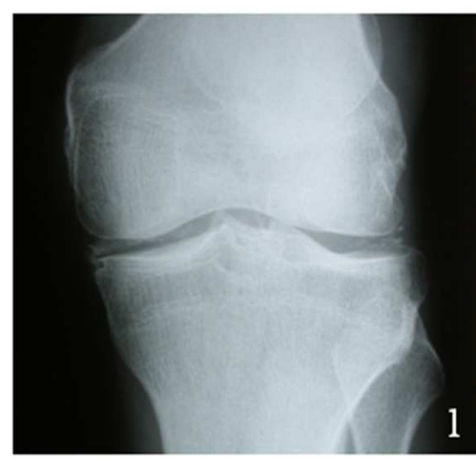

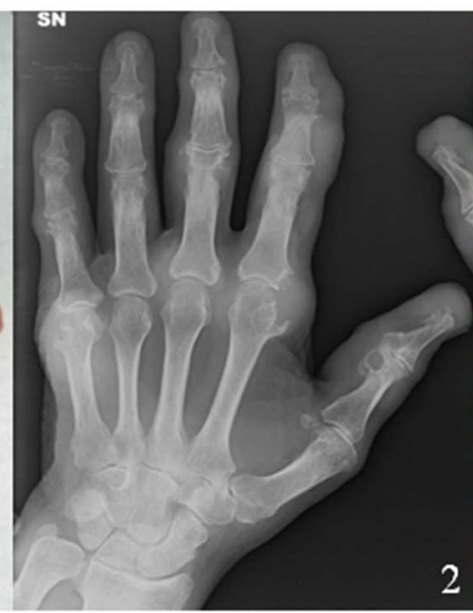

2

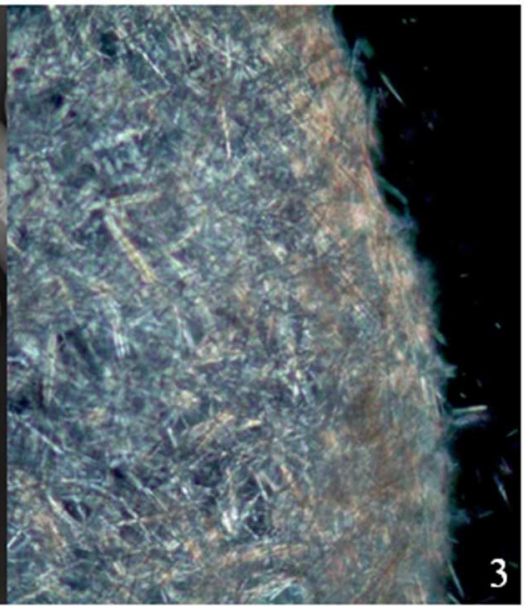

C

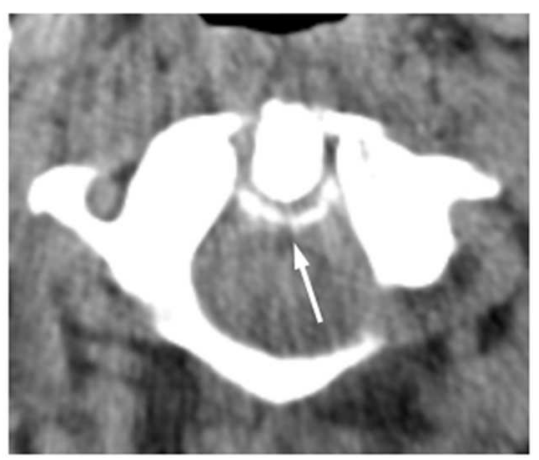

FIGURE 1 | Clinical, radiological and microscopic aspects of gout and calcium pyrophosphate (CPP) crystal deposition. (A) Chronic tophaceous gout (1) with severe joint destructions at radiograph (2) in a male patient with long-standing polyarticular gout. Synovial fluid analysis collected from the $V$ metacarpophalangeal joint showed an aggregate of needle-shape monosodium urate crystals (3). (B) CPP crystal deposition in the knee of a patient with recurrent pseudogout attacks (1). CPP crystals (arrows) observed in synovial fluid under compensated polarized light (2). (C) Axial computed tomography (CT) image of a male patient's cervical spine at C1-C2, illustrating the "crowning" of the dens. Arrow represents CPP deposition. Original figures obtained and reproduced with patients' written informed consent to publication.

New researches on exogenous activators of NLRP3 inflammasome suggest the role of a purine-rich diet, that is observed to increase 5 -fold the risk of an acute attack of gout (22). The exact components of this diet are still debated, but it is known that long-chain (C18) free fatty acids (FFAs) might have a role in macrophage priming. Joosten et al. demonstrated a strong interaction of FFAs with Toll-like receptor (TLR2) that synergized with MSU crystals to induce an ASC/caspase 1-driven IL-1 release (23).

Spikes in systemic levels of a short-chain fatty acid can also contribute to the acute gout flares, as observed by Vieira et al. in a murine model of gout (24). The increase of acetate, which signals through the G protein coupled receptor GPR43, restored inflammation in response to injection of MSU crystals and, in vitro, leads macrophages to produce ROS and assemble the inflammasome.

Endogenous ligands of TLR4 (S100A8/A9) have a role in macrophage priming in gout. Holzinger et al. (25) observed that gouty patients and MSU-injected mice produced high level of this heterodimer and increased IL- $1 \beta$ secretion.

In addition, priming of monocytes of gouty patients by higher levels of soluble uric acid enhances the proinflammatory response upon subsequent exposure to TLR2 and TLR4 agonists (26). There is a concomitant downregulation of IL-1Ra that can be responsible to reinforce the enhanced state of inflammation.

An and colleagues described that C5a potentiates MSU crystals in IL- $1 \beta$ production in a caspase- 1 dependent manner, requiring intracellular calcium mobilization, potassium efflux, and cathepsin B activity (27). P2X7R, a purinergic receptor activated by crystal-triggered ATP, is described to induces potassium efflux, ROS production and activates inflammasome signaling pathway (28).

In the study from Shaw et al. (29), GM-CSF is reported in vitro to differentiate monocytes into inflammatory macrophage, that can express high levels of NLRP3, active IL-1 $\beta$, and active caspase-1, once stimulated with crystals. 


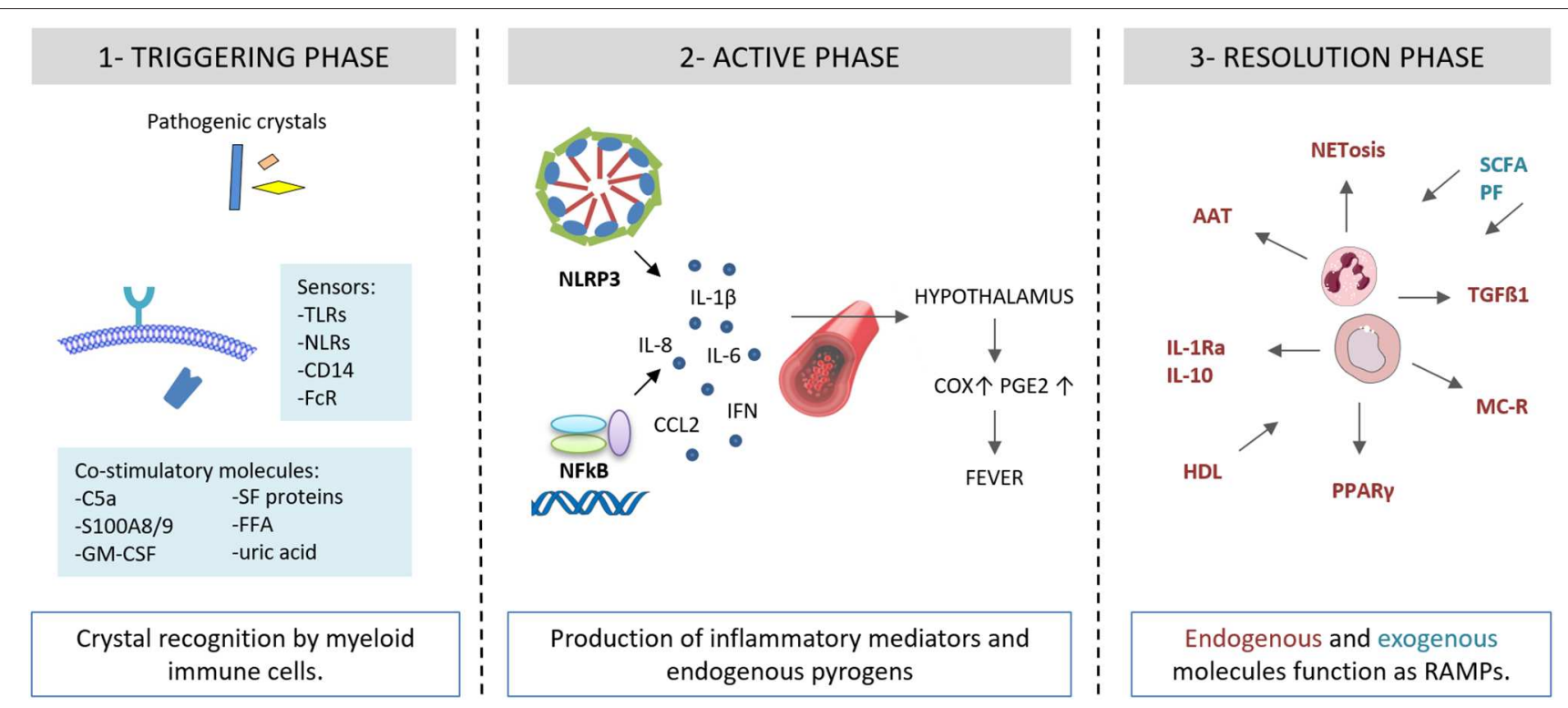

FIGURE 2 | Autoinflammatory steps in crystal-induced inflammation. (1) Crystal deposition triggers the acute attack in presence of co-stimulatory molecules (C5a, S100A8/A9, GM-CSF, SF proteins, FFA, uric acid). Innate immune system receptors (TLRs, NLRs, CD14, FcR) recognize pathogenic crystals promoting acute inflammatory responses in myeloid cells (monocytes/macrophages). (2) Through NLRP3 activation, crystals induce the production of IL-1 $\beta$ and inflammatory mediators and endogenous pyrogens. (3) Endogenous and exogenous molecules function as RAMPs and limit the inflammatory response. AAT, alpha-1-anti-trypsin; C5a, complement factor 5a; CCL2, C-C motif chemokine ligand 2; COX, cycloxygenase; FCR, Fc receptor; GM-CSF, granulocyte-macrophage colony-stimulating factor; granulocyte HDL, high density lipoproteins; IL, interleukin; IL-1Ra, IL-1 receptor antagonist; IFN, interferon; FFA, free fatty acids; NET, neutrophil extracellular traps; MC, melanocortin; NFkB, nuclear factor kB; NLRP3, NACHT-LRRPYD-containing protein-3; NLRs, nod-like receptors, PGE, prostaglandin E; PF, polyphenols; PPAR $\gamma$, peroxisome proliferator-activated receptor $\gamma$; S100A8/A9, S100 calcium-binding protein A8/A9; SCFA, short chain fatty acids; SF, synovial fluid; TGF, transforming growth factor; TLRs, Toll-like receptors.

It is also known that fibrinogen can stimulate cytokine production in macrophages through TLR4 ligation (30). Oliviero et al. (31), priming human THP-1 cells to crystals with low doses of phorbol myristate acetate, reported that fibrinogen is able to increase the inflammatory action of CPP crystals in a dosedependent manner. Later, the same working group demonstrated that protein not lipid fraction of synovial fluid is required for the induction of IL-1 $\beta$ by MSU crystals in macrophages (32). In particular, proteins with molecular weight $>50 \mathrm{kDa}$, such as fibrinogen, can contribute to initiate gouty inflammation.

In this context, it is important to mention that the crystalinduced inflammation, leading to neutrophil migration and accumulation, may be also due to an inflammasome-independent pathway that ensures a strong activation of IL- $1 \beta$ in inflamed tissues, where neutrophils are abundant (33).

\section{THE ROLE OF RESOLUTION-ASSOCIATED MOLECULAR PATTERNS (RAMPS)}

The spontaneous resolution is one of the hallmarks of crystal-induced arthritis. Most of the factors involved in the self-limiting course of the disease have been identified as endogenous molecules that are induced or locally recruited by the inflammatory process itself or are inhibitory proteins normally present in the joint (Figure 2). Among them, transforming growth factor (TGF) $\beta 1$ plays a crucial role in the resolution of crystal-induced inflammation. It has been shown to inhibit leukocyte chemotaxis (34), upregulate the cytokine inducible SH2-containing protein (CIS) (35) and transglutaminase (TG) 2 expression and intracellular negative regulators of cytokines such as the suppressors of cytokine signaling (SOCS)3 (36). Both macrophages (35) and neutrophils (37) are important sources of TGF $\beta 1$.

Similarly to TGF, the natural inhibitor of IL-1 $\beta$, IL-1Ra, and IL-10 might have a key role in the resolution phase as its levels increase in SF of patients with gout (35) and following MSU injection in mice (38).

Lipoproteins have been shown to modulate crystal-induced inflammation through the inhibition of cell activation (39), IL-1 $\beta$ (38) and monocyte/macrophage recruitment (40).

An interesting mechanism of auto-regulation in CIA which is also associated to autoinflammatory syndromes self-resolution is NETosis. It has been observed that MSU crystals induce neutrophil cell death with the release of decondensed nuclear DNA coated with cell granule enzymes to generate neutrophil extracellular traps (NETs) (41). Although NETs have been shown to have both inflammatory and anti-inflammatory effects, NETosis has been supposed to facilitate crystal sequestration in aggregates within tissues limiting the inflammatory response. This process is driven, at least in part, by IL- $1 \beta$ (41).

Interestingly, Apostolidou et al. suggested that the inflammatory attacks of Familial Mediterranean Fever (FMF), could be also triggered by the IL-1 $\beta$ release through NETs. 
Conversely, NETs can serve as inhibitors of NETosis, facilitating the resolution of FMF attacks (42). These observations support a potential role for NET in crystal-induced IL-1ß production and could represents an interesting matter for further studies.

Another event which is associated with CIA resolution is the release of phosphatidylserine-rich microvescicles by infiltrating neutrophils during the inflammatory process. It has been demonstrated that these microvescicles limit inflammasome activation in $\mathrm{C} 5$ a primed macrophages and, as a consequence, IL- $1 \beta$ release $(43)$.

Different other regulatory factors involved in the spontaneous resolution of an acute attack of CIA have been described. Among them, peroxisome proliferator-activated receptor $\gamma$ (PPAR $\gamma$ ) ligands, which reduce the production of IL- $1 \beta$ and TNF induced in vitro by crystals (44) and melacortin receptor (MC-R) agonists which lower the levels of cytokines and polymorphonuclear cell migration in a murine model of MSU crystal-induced peritonitis (45).

The ketone body $\beta$-hydroxybutyrate $(\mathrm{BHB})$ produced in the liver, has been shown to inhibit IL-1 $\beta$ processing in response to MSU crystals by blocking NLRP3 inflammasome and reducing caspase-1 activation (46).

Alpha-1-anti-trypsin (AAT), the major natural inhibitor of serine proteases produced by neutrophils has demonstrated an important inhibitory role in crystal-induced inflammation. AAT not only reduces the conversion of IL- $1 \beta$ precursor into the active cytokine in neutrophils but also increases circulating levels of endogenous IL-1Ra, the IL-1 natural inhibitor (47).

Other factors have recently been described for their effects in modulating the resolution of the acute attack. They are exogenous substances mainly introduced with diet which possess immune-inflammatory-regulatory properties [see review in (48)].

Among them, the most studied compounds are plant polyphenols and short-chain fatty acids.

As far as short-chain fatty acids are concerned, some interesting results have been obtained using butyrate, a major short-chain fatty acid produced during gut flora-mediated degradation of dietary fibers (49). This metabolite has been shown to suppress urate crystal-induced IL-1 $\beta$ production and expression through the specific inhibition of class I histone deacetylase epigenetic enzyme.

Another short-chain fatty acid, acetate, has been recently demonstrated to control the inflammatory response to MSU crystals in mice knee joint by favoring a faster resolution of the inflammatory process (50).

\section{EFFICACY OF IL-1 INHIBITION IN CIA}

Standard treatment for CIA acute attack encompasses corticosteroids, non-steroidal anti-inflammatory drugs (NSAIDs), and colchicine. Besides its capability to block the polymerization of tubulin, colchicine is highly effective at preventing the MSU or CPP crystal-induced processing of proIL$1 \beta$ and the release of IL-1 $\beta$ in monocytes (4) and has a direct effect on IL-1 $\beta$ production in neutrophils (42). Indeed, its use has been extended to a series of autoinflammatory diseases, such as FMF, Behcet's disease and idiopathic recurrent acute pericarditis.

Urate lowering therapies, including allopurinol, febuxostat, probenecid, and lesinurad, are used to prevent MSU crystals formations and gout flares. Among them, febuxostat has recently demonstrated to influence the inflammatory response decreasing IL-1ß serum levels in patients with gout (51).

A significant goal in treating patients intolerant or refractory to traditional drugs has been achieved using IL-1 inhibitors. To date, they include direct inhibitors of IL-1 $\beta$ (canakinumab and gevokizumab), selective inhibitors of the IL-1 receptor (anakinra) and dimeric trap fusion proteins (rilonacept).

The efficacy of anakinra in gout was firstly shown in 2007 by So et al. in a pilot study in which anakinra was successfully administered subcutaneously for 3 consecutive days in 10 patients (8). Later, the same results were observed by Chen et al. (52). Among the 10 patients enrolled, six had a complete response to the drug, three a partial one and one patient had no response. A study on a cohort of hospitalized patients for which standard therapy was ineffective or contraindicated (53) showed an improvement in acute gouty arthritis in the $73 \%$ of the subjects without the onset of notable drug-related adverse events (AEs). A broader multicenter retrospective study on anakinra was carried out in 2013 on 40 patients with an achievement of response in $90 \%$ of the subjects and the finding of AEs due predominantly to infections, appropriately treated with antibiotics. Although there are no available randomized controlled trials (RCTs) to confirm the data from the studies carried out, anakinra seems to be effective in those patients with acute gouty arthritis which do not respond to the standard therapy and for which nonsteroidal antiinflammatory drugs (NSAIDs), corticosteroids (CS) or colchicine are contraindicated.

Few studies have instead investigated the effects of IL-1Ra in pseudogout showing that anakinra was effective and safe in two small series of patients with refractory arthritis due to acute CPP crystal deposition $(9,54,55)$.

The efficacy of rilonacept in gouty arthritis attacks has been investigated in one phase 3 RCT (56) and in three RCTs for the prevention of gout flares during the uric acid lowering therapy. The results of all these studies confirmed the efficacy of the IL1 inhibition in pain improvement and in inflammation markers decrease. However, rilonacept was not approved by EMA nor FDA for these indications so far. In contrast, canakinumab was approved by EMA and FDA in 2013 for the treatment of gout. So and colleagues in 2010 investigated in a phase 2 RCT the dose and efficacy of canakinumab in patients unresponsive to NSAIDs, colchicine or CS. Data showed a significant improvement in pain, swelling and flare recurrence in comparison to subjects treated only with CS (57). In addition, two phase 3 multicenter RCTs carried out on 456 patients (58) achieved the primary endpoint (pain score) better than the control group using CS and the risk of flares was reduced by $62 \%$ over the 12 -week observation period. Of note, more AEs occurred in the canakinumab group in comparison to the control group. The most common ones were infections of the upper respiratory tract, abscesses and gastrointestinal disorders. Similarly, another study by the same authors described the usage of canakinumab in preventing acute 
gout flares after starting allopurinol. As a result, the number of flares was reduced by $72 \%$ in comparison to the group treated with colchicine.

\section{CONCLUSIONS}

Gout and pseudogout are two important and disabling arthropaties with autoinflammatory features. Standard available pharmacological treatments are effective in treating the acute attack in both the diseases. However, while urate-lowering drugs are used to control uricemia and prevent recurrent attacks, no drugs capable of resolving chronic CPP-induced arthritis are currently available. Furthermore, some traditional drugs including allopurinol, corticosteroids, and colchicine are contraindicated in some patients who present drug intolerance or comorbidities such as renal dysfunctions. In those patients, an additional effective way of treatment is needed.

Over the last decade, the advanced understanding of autoinflammatory mechanisms of rare monogenic diseases led to a greater comprehension of the pathogenesis of more common multigenic rheumatic disorders, such as CIA, where innate immunity has demonstrated to play a crucial role. This had an important consequence as new treatment options have been developed for patients affected by those diseases.

\section{REFERENCES}

1. Punzi L, Scanu A, Ramonda R, Oliviero F. Gout as autoinflammatory disease: new mechanisms for more appropriated treatment targets. Autoimmun Rev. (2012) 12:66-71. doi: 10.1016/j.autrev.2012.07.024

2. Masters SL, Simon A, Aksentijevich I, Kastner DL. Horror autoinflammaticus: the molecular pathophysiology of autoinflammatory disease. Annu Rev Immunol. (2009) 27:62168. doi: 10.1146/annurev.immunol.25.022106.141627

3. Shi Y, Evans JE, Rock KL. Molecular identification of a danger signal that alerts the immune system to dying cells. Nature. (2003) 425:5162. doi: 10.1038/nature01991

4. Martinon F, Pétrilli V, Mayor A, Tardivel A, Tschopp J. Gout-associated uric acid crystals activate the NALP3 inflammasome. Nature. (2006) 440:23741. doi: $10.1038 /$ nature 04516

5. Chen CJ, Shi Y, Hearn A, Fitzgerald K, Golenbock D, Reed G, et al. MyD88-dependent IL-1 receptor signaling is essential for gouty inflammation stimulated by monosodium urate crystals. J Clin Invest. (2006) 116:226271. doi: 10.1172/JCI28075

6. Dinarello CA, Ikejima T, Warner SJ, Orencole SF, Lonnemann G, Cannon JG, et al. Interleukin 1 induces interleukin 1. I. Induction of circulating interleukin 1 in rabbits in vivo and in human mononuclear cells in vitro. J Immunol. (1987) 139:1902-10.

7. Dinarello CA. Blocking Interleukin-1 $\beta$ in Acute and Chronic Autoinflammatory Diseases. J Intern Med. (2011) 269:1628. doi: 10.1111/j.1365-2796.2010.02313.x

8. So A, De Smedt T, Revaz S, Tschopp J. A pilot study of IL-1 inhibition by anakinra in acute gout. Arthritis Res Ther. (2007) 9:R28. doi: 10.1186/ ar2143

9. Ottaviani S, et al. Efficacy of anakinra in calcium pyrophosphate crystal-induced arthritis: a report of 16 cases and review of the literature. Joint Bone Spine. (2013) 80:178-82 doi: 10.1016/j.jbspin.2012. 07.018

10. Abhishek A, Doherty M. Update on calcium pyrophosphate deposition. Clin Exp Rheumatol. (2016) 34(4 Suppl 98):32-8.
Another aspect worth to be considered concerns the proinflammatory and damaging role of pathogenic crystals in different body districts. Indeed, cholesterol crystals are associated with vascular endothelial dysfunction and calcium phosphate crystals with vascular calcification.

The demonstration that these crystals trigger the NLRP3 inflammasome and the release of inflammatory cytokines that also drive uric acid crystal-induced inflammation indicates that a drug effective in gout might be relevant in preventing inflammation and limiting injury in other disease including atherosclerosis (59).

In the future, the characterization of new specific molecular pathways in crystal arthritides might offer additional means to treat crystal-related diseases with higher efficacy and specificity.

\section{AUTHOR CONTRIBUTIONS}

All authors listed have made a substantial, direct and intellectual contribution to the work, and approved it for publication.

\section{FUNDING}

This work was supported by Institutional funds from the University of Padova (grant n. DOR1972490/19 to FO and n. DOR1985833/19 to PS).

11. Rosenthal AK, Ryan LM. Calcium pyrophosphate deposition disease. N Engl J Med. (2016) 374:2575-84. doi: 10.1056/NEJMra 1511117

12. Galloway J, Cope AP. The ying and yang of fever in rheumatic disease. Clin Med (Lond). (2015) 15:288-91. doi: 10.7861/clinmedicine. 15-3-288

13. Masuda I, Ishikawa K. Clinical features of pseudogout attack. A survey of 50 cases. Clin Orthop Relat Res. (1988) 229:173-81 doi: 10.1097/00003086-19880400000023

14. Berger RG1, Levitin PM. Febrile presentation of calcium pyrophosphate dihydrate deposition disease. J Rheumatol. (1988) 15:642-3.

15. Sousa APD, Moura C, da Hora PR, Santiago M. Calcium crystal-associated arthropathy mimicking a febrile systemic inflammatory disease in an elderly patient. Oxf Med Case Reports. (2019) 2019:30. doi: 10.1093/omcr/ omz030

16. Shah D, Mohan G, Flueckiger P, Corrigan F, Conn D. Polyarticular gout flare masquerading as sepsis. Am J Med. (2015) 128:e112. doi: 10.1016/j.amjmed.2014.12.025

17. Goh CL, Lai FYX, Chee A, Junckerstorff R. Back pain and fever: when the diagnosis becomes crystal clear. Intern Med J. (2018) 48:48081. doi: $10.1111 /$ imj. 13755

18. Ledingham D, Cappelen-Smith C, Cordato D. Crowned dens syndrome. Pract Neurol. (2018) 18:57-9. doi: 10.1136/practneurol-2017-001793

19. Dalbeth N, Merriman TR, Stamp LK. Gout. Lancet. (2016) 388:203952. doi: 10.1016/S0140-6736(16)00346-9

20. Malik A, Kanneganti TD. Inflammasome activation and assembly at a glance. J Cell Sci. (2017) 130:3955-63. doi: 10.1242/jcs.207365

21. Yaron JR, Gangaraju S, Rao MY, Kong X, Zhang L, Su F, et al. K(+) regulates $\mathrm{Ca}(2+)$ to drive inflammasome signaling: dynamic visualization of ion flux in live cells. Cell Death Dis. (2015) 6:e1954. doi: 10.1038/cddis. 2015.277

22. Zhang Y, Chen C, Choi H, Chaisson C, Hunter D, Niu J, et al. Purine-rich foods intake and recurrent gout attacks. Ann Rheum Dis. (2012) 71:144853. doi: 10.1136/annrheumdis-2011-201215 
23. Joosten LA, Netea MG, Mylona E, Koenders MI, Malireddi RK, Oosting $\mathrm{M}$, et al. Engagement of fatty acids with Toll-like receptor 2 drives interleukin-1beta production via the ASC/caspase 1 pathway in monosodium urate monohydrate crystal-induced gouty arthritis. Arthritis Rheum. (2010) 62:3237-48. doi: 10.1002/art.27667

24. Vieira AT, Macia L, Galvão I, Martins FS, Canesso MC, Amaral FA, et al. A role for gut microbiota and the metabolite-sensing receptor GPR43 in a murine model of gout. Arthritis Rheumatol. (2015) 67:1646-56. doi: 10.1002/art. 39107

25. Holzinger D, Nippe N, Vogl T, Marketon K, Mysore V, Weinhage T, et al. Myeloid-related proteins 8 and 14 contribute to monosodium urate monohydrate crystal-induced inflammation in gout. Arthritis Rheumatol. (2014) 66:1327-39. doi: 10.1002/art.38369

26. Crişan TO, Cleophas MC, Oosting M, Lemmers H, Toenhake-Dijkstra H, Netea MG, et al. Soluble uric acid primes TLR-induced proinflammatory cytokine production by human primary cells via inhibition of IL1Ra. Ann Rheum Dis. (2016) 75:755-62. doi: 10.1136/annrheumdis-2014206564

27. An LL, Mehta P, Xu L, Turman S, Reimer T, Naiman B, et al. Complement C5a potentiates uric acid crystal-induced IL $1 \beta$ production. Eur J Immunol. (2014) 44:3669-79. doi: 10.1002/eji.201444560

28. Gicquel T, Le Daré B, Boichot E, Lagente V. Purinergic receptors: new targets for the treatment of gout and fibrosis. Fundam Clin Pharmacol. (2017) 31:136-46. doi: 10.1111/fcp.12256

29. Shaw OM, Steiger S, Liu X, Hamilton JA, Harper JL. Brief report: Granulocyte-macrophage colony-stimulating factor drives monosodium urate monohydrate crystal-induced inflammatory macrophage differentiation and NLRP3 inflammasome up-regulation in an in vivo mouse model. Arthritis Rheumatol. (2014) 66:2423-8. doi: 10.1002/art.38730

30. Smiley ST, King JA, Hancock WW. Fibrinogen stimulates macrophage chemokine secretion through toll-like receptor 4. J Immunol. (2001) 167:2887-94. doi: 10.4049/jimmunol.167.5.2887

31. Oliviero F, Scanu A, Dayer JM, Fiocco U, Sfriso P, Punzi L. Response to 'Plasma proteins present in osteoarthritic synovial fluid can stimulate cytokine production via Toll-like receptor 4'. Arthritis Res Ther. (2012) 14:405; author reply 406. doi: 10.1186/ar3863

32. Scanu A, Oliviero F, Gruaz L, Galozzi P, Luisetto R, Ramonda R, et al. Synovial fluid proteins are required for the induction of interleukin-1 $\beta$ production by monosodium urate crystals. Scand J Rheumatol. (2016) 45:38493. doi: 10.3109/03009742.2015.1124452

33. Schett G, Dayer JM, Manger B. Interleukin-1 function and role in rheumatic disease. Nat Rev Rheumatol. (2016) 12:14-24. doi: 10.1038/nrrheum. 2016.166

34. Lioté F, Prudhommeaux F, Schiltz C, Champy R, Herbelin A, Ortiz-Bravo $\mathrm{E}$, et al. Inhibition and prevention of monosodium urate monohydrate crystal-induced acute inflammation in vivo by transforming growth factor beta1. Arthritis Rheum. (1996) 39:1192-8 doi: 10.1002/art.1780 390718

35. Chen YH, Hsieh SC, Chen WY, Li KJ, Wu CH, Wu PC, et al. Spontaneous resolution of acute gouty arthritis is associated with rapid induction of the anti-inflammatory factors TGF $\beta 1$, IL-10 and soluble TNF receptors and the intracellular cytokine negative regulators CIS and SOCS3. Ann Rheum Dis. (2011) 70:1655-63. doi: 10.1136/ard.2010.145821

36. Yen JH, Lin LC, Chen MC, Sarang Z, Leong PY, Chang IC, et al. The metastatic tumor antigen 1-transglutaminase-2 pathway is involved in self-limitation of monosodium urate crystal-induced inflammation by upregulating TGF- $\beta 1$. Arthritis Res Ther. (2015) 17:65. doi: 10.1186/s13075-0150592-7

37. Steiger S1, Harper JL. Neutrophil cannibalism triggers transforming growth factor $\beta 1$ production and self regulation of neutrophil inflammatory function in monosodium urate monohydrate crystal-induced inflammation in mice. Arthritis Rheum. (2013) 65:815-23. doi: 10.1002/art.37822

38. Scanu A, Luisetto R, Oliviero F, Gruaz L, Sfriso P, Burger D, et al. High-density lipoproteins inhibit urate crystal-induced inflammation in mice. Ann Rheum Dis. (2015) 74:587-94. doi: 10.1136/annrheumdis-2013-203803

39. Terkeltaub RA, Dyer CA, Martin J, Curtiss LK. Apolipoprotein (apo) E inhibits the capacity of monosodium urate crystals to stimulate neutrophils. Characterization of intraarticular apo $\mathrm{E}$ and demonstration of apo $\mathrm{E}$ binding to urate crystals in vivo. J Clin Invest. (1991) 87:20-6. doi: 10.1172/JCI 114971

40. Scanu A, Oliviero F, Gruaz L, Sfriso P, Pozzuoli A, Frezzato F, et al. Highdensity lipoproteins downregulate CCL2 production in human fibroblastlike synoviocytes stimulated by urate crystals. Arthritis Res Ther. (2010) 12:R23. doi: 10.1186/ar2930

41. Mitroulis I, Kambas K, Chrysanthopoulou A, Skendros P, Apostolidou E, Kourtzelis I, et al. Neutrophil extracellular trap formation is associated with IL-1 $\beta$ and autophagy-related signaling in gout. PLoS ONE. (2011) 6:e29318. doi: 10.1371/journal.pone.0029318

42. Apostolidou E, Skendros P, Kambas K, Mitroulis I, Konstantinidis T, Chrysanthopoulou A, et al. Neutrophil extracellular traps regulate IL-1 $\beta$ mediated inflammation in familial Mediterranean fever. Ann Rheum Dis. (2016) 75:269-77. doi: 10.1136/annrheumdis-2014-205958

43. Cumpelik A, Ankli B, Zecher D, Schifferli JA. Neutrophil microvesicles resolve gout by inhibiting C5a-mediated priming of the inflammasome. Ann Rheum Dis. (2016) 75:1236-45. doi: 10.1136/annrheumdis-2015-207338

44. Akahoshi T, Namai R, Murakami Y, Watanabe M, Matsui T, Nishimura A, et al. Rapid induction of peroxisome proliferator-activated receptor gamma expression in human monocytes by monosodium urate monohydrate crystals. Arthritis Rheum. (2003) 48:231-9. doi: 10.1002/art.10709

45. Getting SJ, Lam CW, Chen AS, Grieco P, Perretti M. Melanocortin 3 receptors control crystal-induced inflammation. FASEB J. (2006) 20:223441. doi: 10.1096/fj.06-6339com

46. Youm YH, Nguyen KY, Grant RW, Goldberg EL, Bodogai M, Kim D, et al. Ketone body $\beta$-hydroxybutyrate blocks the NLRP3 inflammasomemediated inflammatory disease. Nat Med. (2015) 21:263-9. doi: 10.1038/ nm.3804

47. Joosten LA, Crisan TO, Azam T, Cleophas MC, Koenders MI, van de Veerdonk FL, et al. Alpha-1-anti-trypsin-Fc fusion protein ameliorates gouty arthritis by reducing release and extracellular processing of IL-1 $\beta$ and by the induction of endogenous IL-1Ra. Ann Rheum Dis. (2016) 75:121927. doi: 10.1136/annrheumdis-2014-206966

48. Oliviero F, Scanu A. How Factors Involved in the Resolution of Crystal-Induced Inflammation Target IL-1 $\beta$. Front Pharmacol. (2017) 8:164. doi: 10.3389/fphar.2017.00164

49. Cleophas MC, Crisan TO, Lemmers H, Toenhake-Dijkstra H, Fossati G, Jansen TL, et al. Suppression of monosodium urate crystal-induced cytokine production by butyrate is mediated by the inhibition of class I histone deacetylases. Ann Rheum Dis. (2016) 75:593-600. doi: 10.1136/annrheumdis-2014206258

50. Vieira AT, Galvão I, Macia LM, Sernaglia ÉM, Vinolo MAR, Garcia CC, et al. Dietary fiber and the short-chain fatty acid acetate promote resolution of neutrophilic inflammation in a model of gout in mice. J Leukoc Biol. (2017) 101:275-84. doi: 10.1189/jlb.3A1015-453RRR

51. Hao G, Duan W, Sun J, Liu J, Peng B. Effects of febuxostat on serum cytokines IL-1, IL-4, IL-6, IL-8, TNF- $\alpha$ and COX-2. Exp Ther Med. (2019) 17:812-6. doi: 10.3892/etm.2018.6972

52. Chen K, Fields T, Mancuso CA, Bass AR, Vasanth L. Anakinra's efficacy is variable in refractory gout: report of ten cases. Semin Arthritis Rheum. (2010) 40:210-4. doi: 10.1016/j.semarthrit.2010.03.001

53. Ghosh P, Cho M, Rawat G, Simkin PA, Gardner GC. Treatment of acute gouty arthritis in complex hospitalized patients with anakinra. Arthritis Care Res. (2013) 65:1381-4. doi: 10.1002/acr.21989

54. Moltó A, Ea HK, Richette P, Bardin T, Lioté F. Efficacy of anakinra for refractory acute calcium pyrophosphate crystal arthritis. Joint Bone Spine. (2012) 79:621-3 doi: 10.1016/j.jbspin.2012.01.010

55. Diamantopoulos AP, Brodin C, Hetland H, Haugeberg G. Interleukin $1 \beta$ blockade improves signs and symptoms of chronic calcium pyrophosphate crystal arthritis resistant to treatment. J Clin Rheumatol. (2012) 18:3101. doi: 10.1097/RHU.0b013e31826149a2

56. Terkeltaub RA, Schumacher HR, Carter JD, Baraf HS, Evans RR, Wang J, et al. Rilonacept in the treatment of acute gouty arthritis: a randomized, controlled clinical trial using indomethacin as the active comparator. Arthritis Res Ther. (2013) 15:R25. doi: 10.1186/ar4159

57. So A, De Meulemeester M, Pikhlak A, Yücel AE, Richard D, Murphy V, et al. Canakinumab for the treatment of acute flares in difficult-to-treat gouty 
arthritis: Results of a multicenter, phase II, dose-ranging study. Arthritis Rheum. (2010) 62:3064-76. doi: 10.1002/art.27600

58. Schlesinger N, Alten RE, Bardin T, Schumacher HR, Bloch M, Gimona A, et al. Canakinumab for acute gouty arthritis in patients with limited treatment options: results from two randomised, multicentre, active-controlled, double-blind trials and their initial extensions. Ann Rheum Dis. (2012) 71:1839-48. doi: 10.1136/annrheumdis-2011200908

59. Nidorf SM, Thompson PL. Why Colchicine Should Be Considered for Secondary Prevention of Atherosclerosis: An Overview. Clin Ther. (2019) 41:41-8. doi: 10.1016/j.clinthera.2018.11.016
Conflict of Interest: The authors declare that the research was conducted in the absence of any commercial or financial relationships that could be construed as a potential conflict of interest.

Copyright (c) 2020 Oliviero, Bindoli, Scanu, Feist, Doria, Galozzi and Sfriso. This is an open-access article distributed under the terms of the Creative Commons Attribution License (CC BY). The use, distribution or reproduction in other forums is permitted, provided the original author(s) and the copyright owner(s) are credited and that the original publication in this journal is cited, in accordance with accepted academic practice. No use, distribution or reproduction is permitted which does not comply with these terms. 